\title{
Historia de una travesía inconclusa: la comunicación para el desarrollo y el cambio social en la investigación y la docencia universitarias en España
}

\author{
Ana FERNÁNDEZ VISO*
}

Propuesto: 17 de marzo de 2012

Evaluado: 2 de abril de 2012

Aceptado: 10 de abril de 2012

(Abstracts y palabras clave al final del texto)

\section{INTRODUCCIÓN: OBJETO, OBJETIVOS Y METODOLOGÍA}

La comunicación para el desarrollo surge y evoluciona como un campo específico de investigación dentro del marco de los estudios de comunicación a partir de los años cincuenta del siglo XX, vinculada a la creciente actividad de la cooperación internacional al desarrollo y a las tempranas críticas que despertó entre los intelectuales del Sur ${ }^{1}$ (Melkote, 1991; Servaes, 1999; Beltrán, 2005; Mefalopulos, 2008). Inicialmente, se limitó al análisis y el fomento del papel de los medios de comunicación como inductores de modernización y desarrollo y como vehículos difusores de innovaciones occidentales a las poblaciones del entonces bautizado como "Tercer Mundo". De este ámbito específico de reflexión emerge en los años setenta el debate internacional sobre la necesidad de un Nuevo Orden Mundial de la Información y la Comunicación (NOMIC), requisito esencial para la implementación, a su vez, de la receta de desarrollo que entonces reclamaban en el seno de las Naciones Unidas los gobiernos del Tercer Mundo: un Nuevo Orden Económico Internacional (NOEI), más equilibrado e igualitario.

En el contexto de la lucha simbólica por resignificar el concepto de desarrollo como un cambio estructural (social, económico, cultural y político) se gesta la denominación de "comunicación para el cambio social", en tanto propuesta de "otra comunicación" para "otro desarrollo" (Alfaro, 1993) y denuncia de las limi-

\footnotetext{
* Profesora del departamento de Medios, Comunicación y Cultura de la Universidad Autónoma de Barcelona e investigadora del Instituto de la Comunicación de la UAB (InCom-UAB).

1 Especial importancia tuvieron, en este sentido, los pioneros cuestionamientos realizados desde América Latina tanto a las estrategias de desarrollo económico inspiradas en premisas de la economía neoclásica (críticas realizadas en el marco de los trabajos de la CEPAL y que se plasmaron en las premisas del estructuralismo, primero, y de la teoría de la dependencia, a partir de mediados de los años 1960), como a las estrategias modernizadora y difusionista de comunicación para el desarrollo, de raíz funcionalista.
} 
taciones de la behaviour change communication (BCC), predominante en las políticas de las agencias internacionales de cooperación al desarrollo. Inspirada en iniciativas de comunicación emancipatoria de las propias comunidades del Sur, la nueva aproximación a la articulación entre comunicación y desarrollo partía de la convicción de la naturaleza intrínsecamente dialógica, participativa y democratizadora de los fenómenos comunicativos con potencial para generar mejoras sustanciales en las condiciones y las oportunidades de vida de las personas (Gumucio-Dagrón y Tufte, 2008).

La dimensión política que llevaba aparejada ese desplazamiento de perspectiva permitió, al mismo tiempo, trascender definitivamente la cartografía geográfica y epistemológica inicial de esta disciplina, para dar cabida en ella, también, al estudio de las estrategias y las prácticas comunicativas ciudadanas en pro de objetivos de justicia y cambio social y medioambiental. De este modo, bajo el paraguas genérico de "comunicación para el desarrollo y el cambio social" conviven en la actualidad enfoques teóricos, metodológicos e, incluso, axiológicos tan diversos como el marketing social, la comunicación para el cambio de comportamientos individuales, el eduentretenimiento, el advocacy o la movilización social, por mencionar alguno de ellos.

Razones de índole histórico-política y factores contextuales explican, como se verá más adelante, la tardía recepción en España, ya en los años ochenta, de los estudios de comunicación para el desarrollo, así como la marginal atención que les ha prestado nuestra academia hasta la primera década de los años 2000. El presente artículo se propone ofrecer una visión panorámica e histórica de su presencia en la investigación y la docencia universitarias en nuestro país, a partir de una periodización exploratoria en tres etapas diferenciadas en función del nivel de evolución de estos estudios en la comunidad académica española: a) primeras aproximaciones epistemológicas (décadas de 1970 y 1980); b) emergencia de la investigación endógena (década de 1990); y c) bases para la institucionalización del campo (década de 2000).

Más allá de referir, sin ánimo de exhaustividad —un propósito que supera con creces el objetivo de esta primera aproximación a la historia de este campo de estudios en España-, iniciativas de investigación, producción científica y oferta docente relacionadas con la comunicación para el desarrollo y el cambio social en cada uno de estos períodos, se examinan las circunstancias históricas y las condiciones que han ido marcando el desarrollo de este campo disciplinar. Se pretende aportar, además, algunos elementos interpretativos que ayuden a entender el alcance de ese proceso en cada etapa y el grado de interés de nuestra comunidad científica por este ámbito de estudios.

La metodología empleada para elaborar esta propuesta de historización se apoya en el uso de técnicas fundamentalmente cualitativas: por un lado, la observación participante de este campo académico por parte de la autora, que tras más de una década de experiencia profesional en el ámbito de la comunicación social y la comunicación para el desarrollo (incluidos 3 años de trabajo en África occidental) inició a finales de 2006 sus estudios doctorales en el programa Comunicación, Cambio Social y Desarrollo de la Universidad Complutense de Madrid, intensificando, 
desde entonces, su observación de este campo disciplinar ${ }^{2}$. Por otro lado, y complementariamente a la labor de revisión bibliográfica que forma parte de ese proceso de formación especializada, se ha acometido un análisis documental tanto de textos académicos y técnicos sobre investigación y docencia en comunicación en España, como de información pública disponible sobre estudios de postgrado en comunicación y/o desarrollo en las universidades españolas. Finalmente, se han realizado consultas por correo electrónico, telefónicas o en persona a miembros de la comunidad científica española, al objeto de confirmar y ampliar la información reunida, contrastar las conclusiones preliminares e identificar documentos y protagonistas adicionales de la historia de la recepción y desarrollo de la comunicación para el de sarrollo y el cambio social en la universidad española.

El marco referencial y contextual de esta propuesta de historización de los estudios de comunicación para el desarrollo y el cambio social en España es, deudor, asimismo, de meta-análisis existentes sobre: a) la historia de la investigación de la comunicación en España (Moragas, 1981, 1988, 1990; Jones, 1998; Rodrigo Alsina, 2001; García Jiménez, 2007; Martínez Nicolás, 2006, 2008; Martínez Nicolás y Saperas Lapiedra, 2011; Castillo y Carretón, 2010; Soriano, 2008), b) la docencia universitaria en comunicación en España (ANECA, 2005; Benavides Delgado, 2008; Rodrigo Alsina y García Jiménez, 2008; Piñuel, 2011) y c) la docencia y la producción científica españolas en algunas áreas específicamente relacionadas con los estudios de comunicación para el desarrollo y el cambio social (Barranquero, 2009; Barranquero y Sáez Baeza, 2010; Sáez Baeza y Barranquero, 2012).

Después de treinta años de lenta, parcial, pero progresiva aproximación a este enfoque teórico y epistemológico, podemos afirmar que los estudios de comunicación para el desarrollo y el cambio social han alcanzado en nuestro país las condiciones para su consolidación y desarrollo. En la última década se han dado pasos importantes hacia su afianzamiento institucional: se han creado grupos de investigación y programas de postgrado especializados, se han defendido las primeras tesis y trabajos de DEA encuadrados en este marco teórico, se ha dedicado un congreso de la AE-IC a este tema y se ha incrementado notablemente la publicación de libros y artículos especializados de autores españoles. Al mismo tiempo, sin embargo, el avance en este proceso requiere hacer frente, necesariamente, a los desafíos epistemológicos, teóricos e, incluso, ontológicos que hoy caracterizan la situación

2 De manera simultánea a una constante tarea de revisión bibliográfica, esta observación participante en España se desarrolla a través de la participación y la colaboración en diversas iniciativas: la red de investigadores del programa de doctorado 276 en "Comunicación, Cambio Social y Desarrollo" de la Universidad Complutense de Madrid, el equipo de investigación del Observatorio de Políticas de Comunicación (OPC) del Instituto de la Comunicación de la Universidad Autónoma de Barcelona (InCom-UAB), la red de investigación en comunicación y desarrollo REAL-CODE —impulsada por las Universidades de Santiago de Compostela, Vigo y A Coruña-, el Grupo de Investigación Compolíticas de la Universidad de Sevilla, congresos científicos (AE-IC, ULEPICC, IAMCR, Orecomm, etc.), seminarios y jornadas especializados (p. ej. Foro Internacional Comunicación, Desarrollo y Cambio Social, Sevilla, marzo 2008; Seminario Comunicación para el Cambio Social: universidad, sociedad civil y medios, Universidad Rey Juan Carlos, noviembre 2011, etc.) y las actividades de la Cátedra UNESCO de Comunicación del InCom-UAB con profesores visitantes especializados en comunicación, desarrollo y cambio social (p. ej. John Downing, Alfonso Gumucio-Dagrón, Clemencia Rodríguez, etc.) 
de este campo disciplinario en España y que se señalan y analizan en el apartado final del artículo.

\section{PRIMERAS APROXIMACIONES EPISTEMOLÓGICAS A LA COMUNICACIÓN PARA EL DESARROLLO (1970s-1980s)}

La tardía recepción y el lento despegue de los estudios de comunicación para el desarrollo y el cambio social en España guardan una relación evidente con el escaso desarrollo del propio campo académico de la comunicación en este país hasta la década de los años 70, fruto, a su vez, de las limitaciones impuestas por un contexto de cuatro décadas de régimen político dictatorial. Si bien el franquismo desplegó una política desarrollista desde finales de los años $50 \mathrm{y}$, de hecho, España fue considerada como país en desarrollo y potencial receptor de ayuda oficial al desarrollo por los organismos económicos internacionales hasta la década de $1980^{3}$, nuestros investigadores no empezarán a interesarse por la contribución de la comunicación y sus medios a los procesos de desarrollo hasta el advenimiento de la democracia 4 .

Siguiendo a Moragas $(1981,1988)$ y Jones $(1988)$, la reducida producción teórica sobre comunicación de los largos años de la dictadura se centró, fundamentalmente, en las actividades periodísticas y propagandísticas, aunque su naturaleza doctrinaria, antes que científica, dificulta su equiparación con la investigación que por entonces se realizaba en otros países occidentales y en América Latina. Paralelamente, la docencia impartida en organismos públicos como las Escuelas Oficiales de Periodismo, Cine, Publicidad y Radiodifusión y Televisión (constituidas, respectivamente, en 1941, 1947, 1964 y 1967) no aspiraba, precisamente, a formar investigadores con inquietudes intelectuales y espíritu crítico, sino profesionales fieles al franquismo.

La aprobación de la Ley General de Educación de 1970 y, en su desarrollo legislativo, del Decreto 2070/19715, de regulación de los estudios universitarios de comunicación social, abren la puerta a la sustitución de las escuelas oficiales por facultades de Ciencias de la Información y, con ello, a la emergencia de una tradición propiamente científica de investigación sobre comunicación en España (Moragas, 1981; Jones, 1988; Rodrigo Alsina, 2001; García Jiménez, 2007).

El curso 1971/72 inician su actividad las Facultades de Ciencias de la Información de las universidades públicas Complutense de Madrid (UCM) y Autónoma de Barcelona (UAB) y se transforma en Facultad el Instituto de

3 En 1980 el Fondo Monetario Internacional situó a España en el grupo de los países industrializados. No obstante, el Banco Mundial mantuvo la consideración de España como país en desarrollo de renta media hasta un año después y el Comité de Ayuda al Desarrollo de la OCDE siguió calificando a nuestro país como potencial receptor de ayuda al desarrollo hasta 1984.

4 Hemos de recordar que, en contraste con la endeble investigación científica en comunicación que se acometía en España durante los años del franquismo, algunos académicos españoles pudieron desarrollar una fructífera labor investigadora en el exilio. Es el caso, por ejemplo, de Jesús M. Aguirre en Venezuela, Eulalio Ferrer en México, Jesús Martín-Barbero en Colombia e Ignacio Ramonet en Francia, entre otros.

5 Decreto 2070/1971, de 13 de agosto (BOE de 14 de septiembre). 
Periodismo de la universidad privada de Navarra (UN), fundando en 1958. Seis años más tarde se crea una unidad de Ciencias de la Información en la Universidad pública del País Vasco (UPV). Con el nacimiento de las facultades universitarias se produce un destacado avance en la enseñanza y la investigación, en un contexto marcado, además, por el ocaso de la dictadura y la relajación de los controles intelectuales. La promoción de los estudios de comunicación al rango universitario genera, al mismo tiempo, "una estructura de oportunidades para la profesionalización académica en este campo, con unos mecanismos de acceso, consolidación y promoción profesionales que exigen, necesariamente, comprometerse de forma activa en la investigación científica" (Martínez Nicolás, 2008, p. 7).

Esas primeras facultades de comunicación agrupaban a mediados de los años setenta a entre 4.000 y 5.000 estudiantes y a unos quinientos docentes (Jones, 1998, p. 5). La posible aproximación a la comunicación para el desarrollo y el cambio social de esta incipiente comunidad científica se veía dificultada, a priori, por el perfil epistemológico del profesorado, que, como señala Martínez Nicolás (2008, p. 8), procedía en su mayoría de las viejas escuelas oficiales, del mundo profesional de la comunicación social (periodistas, especialistas en radio y televisión, publicistas, etc.) o del ámbito de la formación superior en Humanidades (filología, filosofía y letras, historia, lingüística, etc.). Fue muy escasa la presencia inicial en estas facultades de investigadores formados en la tradición de las ciencias sociales clásicas: sociología, ciencia política, economía, pedagogía, psicología, antropología, etc., perfiles de especialización mucho más afines a los estudios sobre la articulación entre comunicación, cambio social y desarrollo.

De este modo, concluye Martínez Nicolás (2006, p. 146), la investigación comunicativa española arranca esos años prácticamente al margen del fecundo proceso de renovación de los estudios sobre comunicación liderado fuera de nuestro país por las ciencias sociales y que se plasmaba en propuestas epistemológicas de corte crítico, como la economía política de la comunicación y los estudios culturales, y en la recuperación de las sociologías interpretativas. Estas corrientes de pensamiento, de suma importancia para el proceso ya referido de cambio de paradigma en el ámbito de los estudios de comunicación para el desarrollo (Fernández Viso, 2008), llegarán finalmente a nuestro país de la mano de la apertura científica que se inicia por la necesidad de dotar a los estudios de comunicación de referentes científicos y de dignidad académica.

A mediados de la década los investigadores españoles se disponen a entrar en contacto con la comunidad científica internacional, a través de su participación en congresos, foros y organizaciones internacionales de investigadores y de la realización de estancias de investigación en el extranjero. En este sentido, la asistencia de dos profesores de la UAB, Miquel de Moragas y Manuel Parés, a la X Conferencia de la AIERI-IAMCR 6 , celebrada en 1976 en Leicester (Reino Unido), estimulará la recepción y difusión en España de los planteamientos latinoamericanos sobre políti-

6 AIERI: Association International des Etudes et Recherches sur l'Information; en inglés IAMCR: International Association for Mass Communication Research. La AIERI se creó en 1957 en París, impulsada por la UNESCO. 
cas de comunicación e imperialismo cultural, formulados en el marco de una evaluación crítica tanto de la contribución de los medios de comunicación de masas al desarrollo de sus países, como de las premisas de la propia mass communication research. Eran tiempos de debate en torno al NOMIC y, junto a la investigación latinoamericana, tienen gran protagonismo en ese encuentro las tesis de economía política de la comunicación abanderadas por el Center for Mass Communication Research de Leicester (James Halloran, Graham Murdock, Peter Holding, etc.) - anfitrión de la conferencia- y por autores como Kaarle Nordenstreng, Cees Hamelink, Dallas Smythe y Herbert Schiller, entre otros.

La muerte de Franco en 1975 acelera la descomposición del régimen y precipita la transición democrática. Cataluña se plantea esos años su reconstrucción nacional y la recuperación de su lengua y de su cultura, así como el papel de los medios de comunicación en estos procesos. Era, por tanto, un terreno abonado para el de sarrollo de una destacada tradición de investigación sobre estructura y políticas de la comunicación, como, efectivamente, ocurrió 7 . De regreso en Barcelona, Moragas y Parés incorporan el enfoque latinoamericano de la economía política de la comunicación en la actividad docente e investigadora del departamento de Teoría de la Comunicación de la UAB.

Al mismo tiempo, Moragas emprende una señalada labor de recopilación y difusión teórica, con la finalidad de fundamentar el estudio científico y la enseñaza universitaria de la comunicación en nuestro país. En sus primeras obras de divulgación $(1981,1985)$ ya incluye el pensamiento latinoamericano. Los lazos establecidos en Leicester con la academia latinoamericana, a través de la figura de Luís Ramiro Beltrán, se amplían y consolidan en la XII Conferencia de la AIERI-IAMCR, celebrada en Caracas en 1980.

La recuperación de la libertad impulsa la transformación del sistema cultural y comunicativo español, lentamente iniciada en los años 60, con la implantación masiva de la televisión y el desarrollo de la publicidad comercial, y favorecida por la aparición de medios privados reformistas entre 1969 y 1973. Actores dominantes en la estructura de comunicación social franquista desaparecen o pierden importancia, mientras eclosionan en el mapa comunicativo nuevas empresas, grupos, instituciones públicas e, incluso, iniciativas ciudadanas (p. ej. prensa y radios libres) que responden a la demanda de pluralismo informativo y cultural de la sociedad española. En 1983 la Ley del Tercer Canal pone punto final al monopolio televisivo estatal, dando pleno reconocimiento jurídico a la creación de radiotelevisiones públicas en las comunidades autónomas ${ }^{8}$. Seis años más tarde el mercado televisivo se abrirá a los operadores privados.

En estos años de transición, la vertiginosa reconfiguración del mapa mediático español, la recuperación de espacios políticos y comunicativos regionales, locales y comunitarios, la creciente demanda de profesionales de los medios y el interés por

\footnotetext{
7 Algunos autores (Corominas, 1997; Jones, 1998; Gifreu, 2005) denominan Escuela Catalana de Comunicación al nutrido grupo de investigadores vinculados a universidades catalanas que a lo largo de las últimas tres décadas se han dedicado al estudio de la estructura y de las políticas de comunicación.

8 Las televisiones autonómicas vasca y catalana iniciaron sus emisiones antes de la aprobación de la ley.
} 
la deontología periodística propiciarán un acusado mediocentrismo en la investigación sobre comunicación en España, con el consiguiente descuido de las facetas relacional, cultural y simbólica de la comunicación, que fuera de nuestro país acaparaban la atención de la corriente crítica y renovadora de la comunicación para el cambio social.

Ya en los ochenta la academia española se interesó por un amplia variedad de perspectivas y fenómenos comunicativos (Jones, 1998, pp. 13-16) y aunque, en general, manifestó un interés residual - cuando no nulo- por el campo de estudios específico de la comunicación para el desarrollo y el cambio social, algunas líneas incipientes de investigación se encontraban directa o indirectamente muy relacionadas con el análisis de la articulación entre comunicación, desarrollo y cambio social. Cabe citar entre ellas:

1) Los estudios sobre comunicación internacional, estimulados, sobre todo, por los debates en torno al NOMIC y al Informe MacBride concitaron la atención, entre otros investigadores, de Esteban López-Escobar y Pedro Lozano Bartolozzi en la UN, Sara Núñez de Prado y Antonio Sánchez-Bravo en la UCM y Manuel Parés, Josep Gifreu, Mar de Fontcuberta y Marcial Murciano en la UAB.

2) El análisis de la estructura y la economía política de la comunicación y la cultura ocupó a autores como Enrique Bustamante, Bernardo Díaz Nosty y Fernando Quirós en la UCM, Rosario de Mateo en la UAB, Juan Carlos Miguel de Bustos y Ramón Zallo en la UPV y Lluís Bonet en la Universidad de Barcelona (UBA).

3) Las obras de teorías y sociología de la comunicación, donde podían tener cabida las perspectivas modernizadora, difusionista, latinoamericana o participativa de la comunicación para el desarrollo. Entre los autores que se hicieron eco de esos postulados destacaron Miquel de Moragas, Manuel Parés, Enric Saperas y Miquel Rodrigo Alsina en la UAB y Manuel Martín Serrano, José Luis Piñuel, Vicente Romano García y Antonio Sánchez Bravo en la UCM, entre otros.

4) Las investigaciones sobre el potencial educador de los medios de comunicación, promovidas, entre otros estudiosos, por Magda Albero, Mar de Fontcuberta y José Manuel Pérez Tornero en la UAB y Agustín García Matilla en la UCM. El nacimiento en 1986 del Grupo Comunicar, impulsado por José Antonio Aguaded, marca un hito determinante en la evolución de esta tradición teórica en España. El grupo se convierte en pocos años en un referente dentro y fuera de nuestras fronteras y comienza a editar la prestigiosa Revista Comunicar.

5) Consecuencia del cruce entre el interés por los espacios y los medios de comunicación regional y local en el nuevo Estado de las autonomía y la emergencia de medios populares - notable, sobre todo, en Cataluña-, se desarrolla en esta década una línea de trabajo que se denominó comunicación de proximidad. En la medida en la que prestó atención a las experiencias y los planteamientos de los medios de comunicación locales, comunitarios y ciudada- 
nos se aproximaba a otro de los pilares de la comunicación para el cambio social: la comunicación alternativa, popular y participativa. Aquí hay que mencionar los trabajos de José Vidal Beneyto, Miquel de Moragas, María Corominas, Emili Prado o Lluís Bassets en Cataluña ${ }^{9}$. Este enfoque, sin embargo, concentró finalmente su atención en el estudio de la estructura de la comunicación en las regiones históricas de ámbito sub-estatal, vinculado, a su vez, a la línea de investigación en políticas de comunicación ya mencionada.

Se ha de matizar, no obstante, que estas investigaciones no se abordaron desde el marco teórico de la comunicación para el desarrollo o el cambio social. Respondían, más bien, a una aproximación intuitiva al mismo, nacida de la inquietud por democratizar las estructuras y los flujos comunicativos del nuevo régimen político. Serán los manuales de teorías y sociología de la comunicación, ya referidos, y algunos análisis de la comunicación internacional (p. ej. Gifreu, 1986; Murciano, 1980; Fontcuberta, 1985) los que den cuenta de la existencia de ese ámbito de estudios.

Algunas de esas líneas de investigación fueron ganando fuerza a lo largo de la década, como ocurrió con la sociología de la comunicación y la estructura y las políticas de la comunicación — particularmente, en Cataluña y en el País Vasco-, mientras que otras perspectivas de trabajo vinculadas, también, a la comunicación para el desarrollo y el cambio social avanzarían con una intensidad mucho menor. Ejemplo de ello son las trayectorias que experimentaron los estudios de comunicación internacional, la comunicación estratégica o la misma comunicación educativa.

El arco de fenómenos comunicativos y enfoques epistemológicos objeto del interés de la investigación en comunicación española se amplía notablemente en la siguiente década, de la mano del despliegue del mapa universitario autonómico y de la creación y consolidación de nuevas facultades de comunicación por todo el territorio nacional. Este hecho contribuirá a paliar, en parte, la concentración de recursos e instituciones de investigación producida, fundamentalmente, en torno a Madrid y Barcelona.

\section{EMERGENCIA DE LA INVESTIGACIÓN ENDÓGENA (1990s)}

En la década de los noventa despega definitivamente la investigación académica sobre comunicación en España (Jones, 1998; Rodrigo Alsina, 2001; García Jiménez, 2007). Se multiplican las tesis defendidas, los trabajos de investigación acometidos, el número de facultades de comunicación y, con ellas, el de docentes e investigadores. A mediados de esta década el número de centros universitarios que impartían estudios de comunicación ascendía a 20. En su conjunto aglutinaban a

9 Entre la producción científica de esta corriente sobresalen: Bassets, De las ondas rojas a las radios libres (1981); Moragas y Corominas, La comunicación local en Cataluña (1975-1988) (1988); Prado, Las radios libres. Teoría y práctica de un movimiento alternativo (1983); y Vidal Beneyto, Alternativas populares a las comunicaciones de masas (1979). 
unos 20.00 estudiantes y 2.000 profesores. La necesidad apremiante de reclutar profesorado para satisfacer este aumento de la oferta de estudios abrió las puertas de la academia a dos perfiles curriculares diferenciados: por un lado, a los licenciados y doctores que desde el primer momento habían optado por hacer carrera académica; y por otro, apunta Martínez Nicolás (2008, p. 13), a profesionales del ámbito de la comunicación social que se sintieron atraídos por este proceso de reclutamiento. Esta dualidad se reproducirá, como veremos, al interior del embrionario campo de los estudios de comunicación para el desarrollo y el cambio social.

El interés académico por el papel de la comunicación en los procesos de desarrollo y cambio social se verá estimulado, en gran medida, por la progresiva actividad desde mediados de los años 80 del naciente sector español de la cooperación al desarrollo. Si bien la Agencia Española de Cooperación Internacional (AECI) no se crea hasta 1988, España canalizaba ayuda con anterioridad, sobre todo, a América Latina, a través de la Secretaría de Estado para la Cooperación Internacional y para Iberoamérica (SECIPI).

Simultáneamente, entre 1985 y 1995 se produce una eclosión de ONG sin precedentes en nuestro país. Estos actores sociales se hacen netamente visibles en el espacio público a partir de la campaña de recogidas de firmas que inician en Octubre de 1992 para pedir que España destinase el 0,7\% de su PIB a la cooperación al desarrollo. Tras extender a todo el país la protesta por la falta de compromiso político con este objetivo, culmina con la entrega de medio millón de firmas en el Congreso de los Diputados en junio de 1994 (Jerez, Sampedro y López Rey, 2008). Al mismo tiempo, las ONG logran introducir las crisis humanitarias de los Grandes Lagos y los Balcanes en las agendas mediática, pública y política, lo que desencadena una inesperada respuesta solidaria masiva por parte de la sociedad española.

La capacidad de movilización de estas entidades alienta el estudio del fenómeno de la sensibilización social en España y del uso que hacen las ONG de la comunicación. En esta línea empieza a trabajar, por ejemplo, Javier Erro en la Universidad pública de Navarra, desde su doble condición de activista y académico. Ariel Jerez, por su parte, estudiará en la facultad de Ciencias Políticas de la UCM la construcción comunicativa del desarrollo y la cooperación en España (2001).

América Latina concentra en esos años los esfuerzos y los recursos de la acción solidaria española y, en particular, Centroamérica, sacudida en la década de los ochenta por intensos conflictos armados, pero escenario, también, de una multitud de iniciativas de comunicación alternativa, popular, conscientizadora y de resiliencia frente al conflicto. Esas experiencias, junto con las prácticas comunicativas participativas que acompañan la desintegración de los regímenes totalitarios del Sur del continente, despiertan la atención de una parte de las ONG con proyectos en la región y pronto se dan a conocer en nuestro país. Surge, entonces, un interés académico por el estudio de estos fenómenos comunicativos, si bien bastante minoritario.

En los años noventa, por otra parte, nacen las primeras cátedras UNESCO de comunicación en las universidades españolas, canales privilegiados para la difusión del conocimiento que se producía fuera de nuestro país. La Cátedra UNESCO de Comunicación de la UAB fue la primera Cátedra UNESCO de Comunicación que se creó en el mundo, impulsada por Manuel Parés — quien la dirigirá durante 20 
años- y Miquel de Moragas. Se crea en el curso 1989/1990, con el propósito de traer a la UAB académicos de renombre internacional que durante un cuatrimestre impartirían docencia en los dos programas de doctorado en comunicación que por entonces ofertaba esta universidad. Además de los cursos de postgrado, los profesores visitantes ofrecían una serie de conferencias abiertas a todo el alumnado, pero, sobre todo, interactuaban activamente con el profesorado de la facultad de comunicación, generando un intercambio de conocimientos muy fructífero, mientras se tejían, a la vez, redes intelectuales.

La mayoría de los catedráticos UNESCO de Comunicación de la UAB de esa década investigaban los medios y los procesos comunicativos desde la óptica de su aportación al desarrollo, la democratización, el cambio social y la construcción de paz. De este modo, la estancia en Barcelona de Elisabeth Fox, José Marques de Melo, Jesús Martín Barbero, Giuseppe Richeri, Gaetan Tremblay, Josep Rota, Tapio Varis, Carlo Marletti y John Sinclair contribuirá decisivamente a divulgar en la UAB premisas y conceptos básicos del campo disciplinar de la comunicación para el de sarrollo y el cambio social. La cátedra se integró en el Instituto de la Comunicación (InCom-UAB) a partir de la fundación de este centro de investigación por Miquel de Moragas en el curso 1997/98.

La UCM, por su parte, crea en 1993 una Cátedra UNESCO de Comunicación para el Desarrollo bajo la dirección de Antonio Sánchez-Bravo. Esta cátedra se orientó al desarrollo de proyectos, asesoría y consultoría a instituciones públicas y privadas en materia de información, comunicación, desarrollo y, sobre todo, educomunicación. Por aquellos años se intensificaban, todavía más, los vínculos del profesorado de la UCM con la comunidad latinoamericana de investigadores: Carmen Caffarel, Francisco Bernete y Vicente Baca promueven la celebración en julio de 1991 del I Encuentro de Almagro sobre comunicación y movimientos sociales en España y América Latina. Esta cita reúne, entre otros académicos, a Jesús Martín Barbero, Javier Esteinou, Rossana Reguillo, Regina Festa, Gonzalo Abril y Tomás Rodríguez-Villasante. Por último, a finales de la década la UCM pone en marcha un título propio de Experto en Información Internacional y Países del Sur, que coordinará el periodista Javier Bernabé.

Así, poco a poco se van incrementando el conocimiento y el interés en la universidad española por enfoques y fenómenos relacionados con la comunicación para el desarrollo y el cambio social. Hacia mediados de la década comienza a trazarse una suave línea diferenciadora entre quienes se acercaban a este campo disciplinar desde una perspectiva más teórica, analítica o, incluso, meta-analítica, y quienes lo hacían desde una posición activista, implicados directamente en iniciativas de comunicación local y ciudadana o de acción social. En el primer grupo se situaría, por ejemplo, Francisco Sierra, que en 1997 defiende su tesis doctoral sobre pedagogía de la comunicación y desarrollo local, mientras que en el segundo emplazaríamos a investigadores como Manuel Chaparro, muy comprometido con la radio local andaluza (tema de su tesis, defendida en 1996), Víctor Marí, que llega a este enfoque desde su trabajo de comunicación educativa en una entidad social de Vallecas y su integración docente en la UNED, Javier Bernabé, desde la práctica periodística, o Javier Erro, centrado en la comunicación de las ONG. 
Junto a las líneas de investigación abiertas en los años ochenta, y que tendrán continuidad en los noventa, la academia española empieza, entonces, a estudiar fenómenos como la comunicación local participativa, el discurso de las ONG y su labor de sensibilización social y los usos y apropiaciones de las nuevas tecnologías de la información y la comunicación (TIC), desde un encuadre teórico de comunicación y cambio social, marcadamente influido por el pensamiento latinoamericano.

Simultáneamente, Tomás Rodríguez-Villasante y su grupo de trabajo en la facultad de Ciencias Políticas y Sociología de la UCM llevan a cabo una labor capital de introducción y enseñanza de la metodología de investigación-acción participativa (IAP), si bien no la enmarcaban en la tradición de los estudios de comunicación para el desarrollo y el cambio social. Es importante señalar que a partir de los años noventa se incrementan las aproximaciones indirectas a esta perspectiva comunicativa desde otras ciencias sociales y, específicamente, desde los estudios de desarrollo, cooperación internacional, innovación democrática, trabajo social y pedagogía crítica.

La década se cierra, por fin, con la publicación de una serie de libros que dan cuenta de la emergencia de una investigación y una reflexión endógenas, que parten ya de premisas específicas de la comunicación para el cambio social: Comunicación e insurgencia. La información y la propaganda en la Guerra de Chiapas (Sierra, 1997), Radio pública local (Chaparro, 1998), Globalización, nuevas tecnologías y comunicación (Marí Sáez, 1999) e Introducción a la teoría de la comunicación educativa (Sierra, 2000). Conviene matizar, no obstante, que se trataba de aproximaciones sectoriales a la comunicación para el desarrollo y el cambio social, es decir, a algunas corrientes y fenómenos vinculados a un campo de estudios de mayor amplitud y trayectoria. Faltaban por abordar, por ejemplo, perspectivas como la antropológica o la psicológica, así como el estudio de la comunicación rural y la intergrupal ${ }^{10}$. No será hasta los años 2000 cuando se acometa en España un trabajo de recopilación y sistematización de las diferentes teorías, paradigmas, enfoques y prácticas de la comunicación para el desarrollo y el cambio social. En definitiva, cuando se hagan una historia y un estado de la cuestión de esta disciplina.

\section{BASES PARA LA INSTITUCIONALIZACIÓN DEL CAMPO (DÉCADA DE 2000)}

En los años 2000 se completa el mapa de las facultades de comunicación del sistema universitario español, constituido en 2006 por 45 centros de estudio (García Jiménez, 2007: 156). Los nuevos docentes y quienes se habían incorporado en la década de los 90 se interesan crecientemente por nuevas perspectivas de investigación de la comunicación. La globalización, la multiculturalidad y la revolución de las TIC atraen la atención, sobre todo, de jóvenes investigadores, pero también, por

10 En su balance de la investigación de la comunicación en España, Daniel Jones incluía, también, la comunicación popular y alternativa entre los objetos de estudio poco abordados a finales del siglo XX en nuestro país (1998, p. 15). 
ejemplo, de quienes venían trabajando en los ámbitos de la educomunicación, la recepción de la comunicación y la formación de la opinión pública. Del mismo modo, movilizaciones sociales masivas como las generadas por el vertido tóxico del buque petrolero Prestige frente a la costa de Galicia en 2002, el rechazo a la guerra de Irak en 2003 y la repulsión tanto por los atentados del 11 de marzo de 2004 en Madrid, como por la manipulación informativa en torno a la autoría de esos hechos, suscitarán un gran interés académico.

Paralelamente, el volumen de la Ayuda Oficial al Desarrollo (AOD) española se dispara a partir de 2003, lo que redunda en un mayor interés de la universidad por la investigación en temas relacionados con el desarrollo y el cambio estructural, por la creación de estudios de postgrado en este ámbito y por el incremento de los programas de cooperación universitaria al desarrollo ${ }^{11}$ (Arias Careaga y Molina Bayón, 2008). El sector no gubernamental crece, también, en el marco de este favorable escenario a su actividad y la AECI impulsa, en desarrollo de su II Plan Director (2005-2008), la elaboración de las estrategias de educación para el desarrollo y de cultura y desarrollo de la cooperación española, procesos liderados por Mari Luz Ortega Carpio y Alfons Martinell, respectivamente.

Las facultades de comunicación pierden definitivamente en el nuevo siglo la exclusividad en la investigación comunicativa en España. Según ha observado Martínez Nicolás (2008, p. 21), disciplinas académicas como la sociología y las ciencias políticas impulsan decisivamente los estudios sobre comunicación política; la psicología social y cognitiva se interesan por los efectos de los medios y por la persuasión; la sociología del consumo y la antropología hacen notables aportaciones a los estudios sobre audiencias y recepción de los medios; la pedagogía está contribuyendo al avance de la investigación sobre comunicación y educación y, finalmente, la economía y la ciencia política han adoptado como objeto de estudio las políticas de comunicación y cultura.

En esta década empiezan a impartirse en España, como veremos más adelante, programas o asignaturas de postgrado sobre comunicación para el desarrollo y el cambio social. En contraste, siguiendo los resultados de una investigación de Barranquero y Sáez (2010), los planes de estudio de las licenciaturas y grados de periodismo, comunicación audiovisual y publicidad y relaciones públicas continúan obviando la existencia de esta perspectiva teórica, hasta el punto de que estos autores no dudan en denunciar una "invisibilización académica de la comunicación alternativa y de la comunicación para el cambio social" (2010, p. 9).

En ese sentido, el proceso de adaptación de los planes de estudio al Espacio Europeo de Educación Superior ha supuesto una oportunidad perdida para integrar esos saberes en los nuevos grados de comunicación ${ }^{12}$. Un resultado previsible, por otra parte, si tenemos en cuenta que entre los conocimientos disciplinares y las com-

11 Las primeras jornadas de cooperación universitaria al desarrollo se celebraron en Madrid en 1987 bajo el título de "Jornadas sobre la Universidad Española ante la Cooperación con los Países en Vías de Desarrollo".

12 Como excepción, hay que mencionar que la Universidad de Cádiz tiene previsto ofertar una asignatura de grado optativa sobre comunicación para el desarrollo y el cambio social a partir del curso 2012-13. 
petencias profesionales propuestos en el Libro Blanco de los Grados de Comunicación (ANECA, 2005) no se contemplaban la perspectiva epistemológica ni las competencias profesionales propias de la comunicación para el desarrollo y el cambio social. El estudio cualitativo sobre el presente y el futuro de la teoría de la comunicación en España presentado por Rodrigo Alsina y García Jiménez (2008) en el congreso fundacional de la Asociación Española de Investigación de la Comunicación (AE-IC) evidenció el desinterés por esta corriente académica de la mayoría ${ }^{13}$ de los teóricos consultados, conclusión que corrobora un reciente estudio sobre la enseñanza y la investigación universitarias en torno a la comunicación en Europa y en América Latina (Piñuel, 2011).

A pesar de ello, en los últimos diez años ha aparecido una nueva generación de investigadores interesados en el análisis de la articulación entre la comunicación social y los procesos de desarrollo y cambio. Este fenómeno se ha hecho manifiestamente visible en el aumento del número de investigaciones doctorales (bien tesis, bien trabajos de DEA) realizadas en torno a temáticas propias de este campo disciplinar. Sólo en lo que a tesis doctorales sobre comunicación alternativa y para el cambio social se refiere, Sáez y Barranquero cifran en 35 los trabajos defendidos entre 2001 y 2011 en universidades españolas, frente a los 5 presentados en la década anterior (2012, pp. 7-10). Se ha abordado, también, una labor de recopilación, meta-análisis y divulgación de las teorías, corrientes y autores que integran el campo de la comunicación para el desarrollo y el cambio social ${ }^{14}$, lo que contribuye a situar la reflexión en torno a las prácticas en una perspectiva más amplia y, sobre todo, histórica. Por último, se ha sucedido la publicación de libros (Sierra, 2002, 2006; Chaparro, 2002; Erro, 2002, 2003; Aparici y Marí, 2003; Marí, 2004, 2011; Miguel de Bustos, 2007; Martínez-Gómez y Lubetkin, 2008, 2010, 2011).

En este punto, nos encontramos con dos líneas divisorias en la investigación que se acomete. La primera se refiere a la actitud epistémica y al nivel de reconocimiento de la genealogía de esta tradición disciplinar. Permitiría diferenciar entre los trabajos que centran su atención en una de las diferentes corrientes que conforman este ámbito disciplinar (por ejemplo, la sensibilización mediática o los estudios sobre medios comunitarios), sin adscribirse ni hacer referencia explícitamente a este marco teórico, y las aproximaciones holísticas y globales al mismo, desde el conocimiento y el análisis de la historia y de las múltiples raíces y dimensiones de la comunicación para el desarrollo y el cambio social.

La segunda línea divisoria se delinea entre quienes presentan un perfil de corte más teórico y reflexivo, interesados en aplicar las herramientas teóricas y conceptuales de las ciencias sociales clásicas y de las humanidades al estudio de los fenó-

13 Solamente uno de los entrevistados incluyó "la comunicación y el desarrollo" en su respuesta a la pregunta de cuáles eran las áreas de la Teoría de la Comunicación española no estudiadas y que deberían serlo (García Jiménez, 2007, p. 275).

14 Por su revisión de un conjunto más o menos amplio de corrientes del paradigma de la comunicación para el desarrollo y el cambio social destacan, entre otros, los trabajos de Del Valle Rojas (2005), Barranquero (2008), Fernández Viso (2008) y Rau de Almeida Callou (2010). A esta labor también han contribuido los números monográficos que publicaciones como la Revista Española de Desarrollo y Cooperación (n. ${ }^{\circ} 15$ y n. $\left.{ }^{\circ} 25\right)$ y Redes.com (n. ${ }^{\circ} 1$ y n. $\left.{ }^{\circ} 4\right)$ han dedicado a esta perspectiva. 
menos comunicativos, y quienes provienen del ámbito laboral de la cooperación al desarrollo, el periodismo y las organizaciones sociales españolas, o, simplemente, se implican más en la práctica y en la acción, colaborando en proyectos de acción social y de desarrollo. Estos investigadores tienen un perfil más profesionalista-activista y, en algunos casos, más intuitivo, podríamos decir, antes que académicocientíficista.

Otro hecho que contribuirá al avance de este ámbito académico en España es el establecimiento de las primeras redes de contactos y de trabajo entre investigadores que comparten el interés por la comunicación para el cambio social ${ }^{15}$. En algunos casos se trata de iniciativas donde convergen académicos y profesionales, como el Foro Comunicación y Ciudadanía que impulsan desde Navarra en 2004 Javier Erro y Teresa Burgui. En otros, son redes y grupos de investigación que se han ido tejiendo en el seno de las facultades de comunicación: Compolíticas (grupo de estudios en comunicación, políticas y cambio social de la Universidad de Sevilla); el grupo de doctorandos y docentes del programa de doctorado 276 en "Comunicación, Cambio Social y Desarrollo" de la UCM; el grupo de Periodismo Preventivo y el grupo de Mediación Dialéctica de la Comunicación Social de la UCM; el núcleo de investigadores del Instituto Interuniversitario de Desarrollo Social y Paz de las universidades de Alicante y Jaume I (IUDSP); la red de trabajo sobre cultura y desarrollo en torno a la Cátedra UNESCO de Políticas Culturales de la Universidad de Girona; o la recientemente creada Red Europa-América Latina de Comunicación y Desarrollo (REAL-CODE), promovida por las universidades de Santiago, Vigo, A Coruña, Extremadura, Salamanca, Sevilla, Rey Juan Carlos de Madrid, UAB y diversas universidades europeas y latinoamericanas ${ }^{16}$.

Al mismo tiempo, se han incrementado los contactos con investigadores internacionales especializados en este campo y aquí hay que destacar, de nuevo, la labor realizada por la Cátedra UNESCO de Comunicación InCom-UAB en los años 2000, al traer a Barcelona a destacados expertos como Armand Mattelart, Thomas Tufte, Rosana Reguillo, Alfonso Gumucio-Dagrón, Clemencia Rodriguez, Rafael Obregón o John Downing, entre otros. Si bien, desde 2008 ya no se trata de estancias de cuatro meses, sino de invitaciones para impartir conferencias.

Finalmente, un paso importante para el reconocimiento institucional de los estudios de comunicación para el desarrollo dentro de la comunidad científica española fue la organización del II congreso de la AE-IC en 2010 bajo el título de "Comunicación y Desarrollo en la era Digital", evento que inauguraron Thomas Tufte y Rafael Obregón, con sendas conferencias sobre comunicación para el cambio social y el desarrollo.

15 Un precedente de estas redes académicas fue la creación en 1994 de ORBICOM, la red que agrupa a las Cátedras UNESCO de Comunicación existentes en todo el mundo y que inicialmente contó con la presencia de las Cátedras de la UCM y de la UAB.

16 Si bien su objeto de estudio es el desarrollo y no la comunicación, no queremos dejar de mencionar la labor de reflexión y difusión realizada, también, por el Instituto Universitario de Estudios sobre Desarrollo y Cooperación Internacional-Hegoa, fruto de un convenido suscrito en 1997 entre la Universidad del País Vasco y la Asociación Hegoa. 
En términos de docencia, los años 2000 han visto nacer los primeros programas de postgrado en comunicación, desarrollo y cambio social. Los pioneros fueron un master en "Comunicación y Desarrollo" y un título de "Experto Universitario en Comunicación y Desarrollo Local" ofertados por la Universidad de Sevilla y el programa de doctorado 276 en "Comunicación, Cambio Social y Desarrollo" de la Universidad Complutense de Madrid. En la actualidad, la UCM oferta un master oficial y un doctorado en "Comunicación Social", con un itinerario de especialización en "Comunicación, Cambio Social y Desarrollo"17 y las universidades Internacional de Andalucía y Pablo de Olavide, un master oficial en "Comunicación y Desarrollo"18. La Universidad Pontifica de Salamanca, por su parte, oferta un magíster en "Comunicación, Cambio Social y Desarrollo".

Otras iniciativas destacadas son el master en "Comunicación con Fines Sociales" de la Universidad de Valladolid (UVa), el master en "Comunicación y Problemas Socioculturales" de la Universidad Rey Juan Carlos de Madrid (URJC), el master en "Mediación Intermediterránea" de la UAB, el master en "Comunicación y Educación en la Red" de la UNED, el master en "Comunicación y Cultura" de la Universidad de Sevilla (US) y los másteres en "Gestión Estratégica e Innovación en Comunicación" e "Investigación en Comunicación Periodística" de la Universidad de Málaga (UMA). Todos ellos cuentan con asignaturas específicas sobre comunicación, desarrollo y/o cambio social, al igual que ocurre con varios programas que se integran en la creciente oferta existente de másteres, magísteres y otros títulos de postgrado en desarrollo, cambio social, paz y resolución de conflictos ${ }^{19}$. Estos últimos, sin embargo, suelen limitar la presencia de contenidos relacionados con la tradición disciplinar de la comunicación para el desarrollo y el cambio social a la comunicación de las ONG, la educación para el desarrollo, el marketing social y las campañas de incidencia política.

En torno a esos programas de postgrado hay un nutrido grupo de profesores que se centran, en su mayoría, en alguna de las corrientes que engloba la comunicación para el desarrollo y el cambio social: Francisco Sierra, Miguel Vázquez Liñán, José M. ${ }^{a}$ León Rubio, Eugenio Antonio Pérez Córdoba y Trinidad Núñez Domínguez (US); Miguel Ángel Sobrino, José Antonio Alcoceba y David Álvarez (UCM);

17 Este programa proviene del referido doctorado 276 que la UCM empezó a ofertar en el curso 2004/2005, impulsado por Miguel Ángel Sobrino.

18 Este programa se inscribe en el marco de una oferta especializada de estudios de postgrado en materia de desarrollo, conformada por un master en "Derechos Humanos, Interculturalidad y Desarrollo" y otro en "Desarrollo Económico y Sostenibilidad". Estos dos programas cuentan con una asignatura sobre "Cultura, comunicación y desarrollo".

19 Entre esos programas especializados que cuentan con asignaturas sobre temáticas propias de la comunicación para el desarrollo destacan los magísteres en "Cooperación Internacional Descentralizada: Paz y Desarrollo" y "Participación y Desarrollo Comunitario" y el master en "Desarrollo y Cooperación Internacional" de la Universidad del País Vasco (UPV); los magísteres en "Investigación Participativa para el Desarrollo Local" y "Cooperación Internacional" y el curso de "Experto en Promoción y Gestión de ONG" de la UCM; el master oficial y doctorado internacional en "Estudios de Paz, Conflictos y Desarrollo" del IUDESP; el master en "Cooperación Internacional para el Desarrollo" de la UVa; el master en "Tecnología para el Desarrollo Humano y la Cooperación" de la Universidad Politécnica de Madrid (UPM); el master en "Cooperación Internacional al Desarrollo" de ICADE; y el master en "Psicología de la Intervención Social Comunitaria" de la US. 
Roberto Aparici y Jesús Prieto (UNED); Ana Teresa López Pastor, Susana de Andrés del Campo, Mari Cruz Alvarado López y Henar Pascual (UVa); Ana Tamarit (UPSA); Manuel Chaparro y Agustín García Matilla (UMA); José Carlos Sendín (URJC); Teresa Velázquez, Olga del Río y Xavier Giró (UAB); Juan Carlos Miguel de Bustos, M. ${ }^{a}$ José Cantalapiedra, Simón Peña y Santiago Pozas (UPV); Eloísa Nos, Sofía Herrero y Eva Espinar (IUDESP); y Rafael Miñano, Susana Muñoz, Marta Ortega, Manuel Sierra, Miguel Ángel Valero, Ana Gómez y Ángel Herranz (UPM).

A esa lista de docentes se suman los nombres de otros investigadores de universidades españolas que trabajan desde una perspectiva explícita ${ }^{20}$ de comunicación para el desarrollo y/o el cambio social, como Isidoro Arroyo (URJC), Alejandro Barranquero (Universidad Carlos III de Madrid), Javier Bernabé (UCM), Florencio Cabello (UMA), Manuel González López (UMA), Ana Fernández Viso (UAB), Hildegart González (Universidad de Navarra), Ariel Jerez (UCM), Víctor Marí (Universidad de Cádiz), Alfons Martinell (Universidad de Girona), Mari Luz Ortega (ETEA), Vanesa Saiz, Marina Mantini y Ana M. ${ }^{a}$ López Cepeda (Universidad de Castilla La Mancha), Víctor Sampedro (URJC) y Víctor Santiago Pozas (UPV).

La investigación sobre comunicación para el desarrollo y el cambio social cuenta en 2012 en España, en conclusión, con notables puntos de apoyo a partir de los que estabilizarse y avanzar.

\section{MIRANDO HACIA ADELANTE: OPORTUNIDADES Y DESAFÍOS DEL CAMPO}

La formación de grupos y redes de investigación, la multiplicación de trabajos doctorales y de bibliografía especializada, la creación de proyectos docentes y el incremento del interés de una parte del profesorado universitario español por la comunicación para el desarrollo y el cambio social permiten constatar que se han dado pasos importantes y esperanzadores hacia la institucionalización de este ámbito disciplinar en España. No obstante, no significa que se haya alcanzado el estadio de masa crítica, parafraseando a Martínez Nicolás (2006). Entre otros motivos, porque esta comunidad científica en ciernes ha de afrontar necesariamente una serie de desafíos epistemológicos, teóricos e, incluso, ontológicos que hoy caracterizan su situación.

En primer lugar, tras las etapas iniciales de aproximación intuitiva, recopilación y divulgación teórica - inacabada-, diseño e implicación, en algunos casos, en proyectos de comunicación para el cambio (social, comunitario, de comportamien-

20 Por motivos obvios de espacio se ha tenido que limitar este segundo listado a profesores universitarios que cuentan con trabajos de investigación explícitamente enmarcados por ellos mismos en la tradición de los estudios de comunicación para el desarrollo y el cambio - antes que en otras líneas de trabajo afines, como la educomunicación, la comunicación local o las políticas de comunicación - o en el estudio de la dimensión comunicativa de procesos de cambio social, como es el caso de Sampedro y Jerez. No obstante, somos conscientes de que son muchos más los investigadores españoles que se han aproximado a este campo de estudios y que habrían de ser incluidos en una descripción más exhaustiva. 
tos individuales, etc.) — bien en España, América Latina o África-, necesitamos desarrollar más investigación empírica, desde la que poder hacer aportaciones propias y valiosas al avance teórico del campo. Ello exige superar el rechazo a teorizar que parece prevalecer en este ámbito (Barranquero, 2009, p. 645), pero, sobre todo, como expresa Martínez Nicolás, "fundamentar la exploración de la realidad en el corpus de los conocimientos - ideas, teorías - disponibles y derivar de ellos hipótesis plausibles que necesariamente deberán ser contrastadas con aquella realidad -números, cuentos: datos-que buscan explicar" (2008, p. 24). En este sentido, se impone resolver la insuficiencia metodológica que varios autores han detectado en la investigación española en comunicación (Castillo y Carretón, 2010, p. 39; Martínez Nicolás y Saperas Lapiedra, 2011, p. 119-125).

Un segundo reto para consolidarse y crecer proviene de la actual situación de dispersión de conocimientos, recursos e investigadores. Resulta esencial aunar el corpus de saberes compartidos, debatir perspectivas e intercambiar inquietudes, para vencer la fragmentación que caracteriza a este campo en nuestro país y estimular el desarrollo de un conocimiento común y colectivamente construido. Del mismo modo, también es importante tender puentes entre los perfiles de carácter más teórico y los de naturaleza predominantemente activista (incluidos los profesionales del sector de la cooperación al desarrollo y la acción social), llamados a complementarse mutuamente. Por último, podemos y debemos avanzar más en el meta-análisis de los resultados de la reflexión y la investigación propias.

En tercer lugar, hemos de asumir, asimismo, con decisión el desafío de desarrollar, debatir y precisar las herramientas conceptuales y metodológicas de nuestro campo de estudios, con el fin de evitar discusiones estériles, imprecisiones y confusiones y poder construir juntos.

Necesitamos, también, ser más explícitos y rigurosos en relación a nuestras teorías del cambio social, sobre todo, porque el diseño o el análisis de cualquier proceso de cambio descansan en la comprensión de cómo funcionan los cambios y qué los produce. Benavides Delgado nos recuerda que la universidad "tiene una especial obligación de hacerse cargo de conceptualizar los cambios y establecer modelos y explicaciones en unas áreas de conocimiento todavía poco definidas y sujetas a unas transformaciones muy vertiginosas y de grandes efectos sociales" $(2008$, p. 176). Debemos ser descriptivos, por tanto, antes de poder ser prescriptivos.

Esa labor pasa, ineludiblemente, por aproximarse a otras ciencias sociales, para desarrollar un marco de análisis capaz de dar cuenta de la complejidad de los procesos de cambio social. Hemos hecho bastante investigación sobre medios, pero muy poca sobre relaciones humanas y procesos comunicativos, y mucha menos, todavía, sobre la interacción entre las diferentes dimensiones y etapas del cambio social -incluyendo su importante dimensión cultural- En este sentido, es primordial impulsar proyectos de investigación de mayor envergadura, interdisciplinares y que aborden la complejidad de los procesos de transformación social, cultural y comunicativa en curso.

Finalmente, la consolidación en España de los estudios de comunicación para el desarrollo y el cambio social encuentra un formidable obstáculo en la crisis económica, que amenaza con cerrar la estructura de oportunidades para la estabilización 
académica de buena parte de los investigadores incorporados en la última década a la universidad. A ello hay que sumar el drástico recorte de las inversiones públicas en investigación y el sesgo en las características de la investigación comunicativa inducido por el sistema de acreditación de los méritos académicos, el "efecto ANECA" (Soriano, 2008).

\section{REFERENCIAS BIBLIOGRÁFICAS}

Alfaro, R. M., 1993. Una comunicación para otro desarrollo. Lima: Asociación de Comunicadores Sociales Calandria.

AnecA, 2005. Libro Blanco de los Títulos de Grado en Comunicación. Disponible en: http://www.aneca.es/media/150336/libroblanco_comunicacion_def.pdf [Última consulta: 25 febrero 2012]

APARICI, R. y MARÍ, V. (coords.), 2003. Cultura popular, industrias culturales y ciberespacio. Madrid: UNED.

Arias Careaga, S. y Molina Bayón, E., 2008. Universidad y Cooperación al Desarrollo. La experiencia de las universidades de la ciudad de Madrid. Madrid: Los Libros de la Catarata.

BARRANQUERO, A., 2008. Latinoamérica en el paradigma participativo de la comunicación para el cambio. Tesis doctoral presentada en el Departamento de Periodismo de la Universidad de Málaga. Málaga: Universidad de Málaga.

— 2009 . The state of the art in citizens' communication for social change in Spain. Development in practice, 19, pp. 643-653.

Barranquero, A. y SÁez Baeza, C., 2010. Comunicación alternativa y comunicación para el cambio social democrático: sujetos y objetos invisibles en la enseñanza de las teorías de la comunicación. Ponencia presentada en el II Congreso Internacional AE-IC 2010 “Comunicación y desarrollo en la era digital”. Málaga, 2-5 de febrero de 2010.

BASSET, L. (ed.), 1981. De las ondas rojas a las radios libres. Barcelona: Gustavo Gili

BELTRÁN, L. R., 2005.: La comunicación para el desarrollo en Latinoamérica: un recuento de medio siglo. Universidad de Buenos Aires, III Congreso Panamericano de la Comunicación, panel 3: Problemática de la comunicación para el desarrollo en el contexto de la sociedad de la información. Buenos Aires, Argentina, 12-16 de julio de 2005. [En línea] Disponible en: http://www.infoamerica.org/teoria_textos/lrb_com_desarrollo.pdf [Última consulta: 25 febrero 2012].

Benavides Delgado, J., 2008. El estado de la comunicación en la enseñanza universitaria: Una reflexión sobre el futuro y el inmediato pasado. En: Separata Revista Comunicación y Pluralismo, pp. 173-194.

CASTILlo, A. y CARRETÓN, M. ${ }^{\text {a }}$ C., 2010. Investigación en comunicación: Estudio bibliométrico de las revistas de comunicación en España. Comunicación y Sociedad, 23 (2), pp. 289-327.

Chaparro, M., 1998. Radio pública local. Madrid: Fragua.

- 2002. Sorprendiendo al futuro. Comunicación para el desarrollo e información audiovisual. Barcelona: Los Libros de la Frontera.

Corominas, M., 1997. Estructura y polítiques de la comunicació. En: J. Berrio (ed.). Un segle de recerca en comunicació a Catalunya. Estudi critic dels principals àmbits d'investigació de la comunicación de massa. Bellaterra: Servei de Publicacions de la Universitat Autònoma de Barcelona, pp. 99-150. 
ERro, J., 2002. Comunicación, desarrollo y ONGD. Bilbao: Hegoa. , 2003. Descubrir y construir procesos de comunicación social. Bilbao: Hegoa.

FERNÁNDEZ ViSO, A., 2008. Genealogía de la comunicación para el desarrollo. Trabajo doctoral para la obtención del Diploma de Suficiencia Investigadora (DEA) del programa de doctorado "Comunicación, Cambio Social y Desarrollo" del Departamento de Sociología IV de la Universidad Complutense de Madrid (UCM). Madrid: UCM.

FontCuBERTA, M. de (dir.), 1985. La comunicación internacional. Barcelona: Mitre.

GARCÍA JimÉNEZ, L., 2007. Las teorías de la comunicación en España: un mapa sobre el territorio de nuestra investigación (1980-2006). Madrid: Tecnos.

Gifreu, J., 1986. El Debate internacional de la comunicación. Barcelona: Ariel.

$\longrightarrow$, 2005. El Informe MacBride desde Cataluña: balance de una esperanza. Quaderns del CAC, 21 (enero-abril), pp. $79-81$.

Gumucio-Dagrón, A. y Tufte, T. (Comps.), 2008. Antología de comunicación para el cambio social: lecturas históricas y contemporáneas. South Orange (New Jersey); La Paz (Bolivia): Consorcio de Comunicación para el Cambio Social/ Plural Editores.

JEREZ, A., 2001. La construcción comunicativa del desarrollo y la cooperación en España (1994-2000): la disputa discursiva entre las ONG, los movimientos sociales y los medios de comunicación. Tesis doctoral presentada en el Departamento de Ciencia Política y de la Administración II de la Universidad Complutense de Madrid. Madrid: UCM.

Jerez, A.; SAMPedro, V. y LóPez Rey, J. A., 2008. Del 0'7\% a la desobediencia civil: política e información del movimiento y las ONG de desarrollo (1994-2000). Madrid: Centro de Investigaciones Sociológicas.

JONES, D., 1998. Investigación sobre comunicación en España: evolución y perspectivas. Zer, 5 (3), [versión en línea] pp. 1- 24 [versión en papel, pp. 13-51]. Disponible en: http://www.ehu.es/zer/es/hemeroteca/articulo/investigacion-sobre-comunicacion-enespana-evolucion-y-perspectivas/58 [Última consulta: 2 marzo 2012].

MARÍ SÁEZ, V., 1999. Globalización, nuevas tecnologías y comunicación. Madrid: Ediciones de la Torre.

$\longrightarrow$, (coord.), 2004. La red es de todos: cuando los movimientos sociales se apropian de la red. Madrid: Editorial Popular.

- 2011. Comunicar para transformar, transformar para comunicar. Tecnologías de la información desde una perspectiva de cambio social. Madrid: Editorial Popular.

Martínez Gómez, R. y LuBETKIn, M. (eds.), 2008. Comunicación y desarrollo: en busca de la coherencia. Madrid: Fundación Carolina y Siglo XXI.

$\longrightarrow$, (coords.), 2010. Comunicación y desarrollo: pasos hacia la coherencia. Zamora: Comunicación Social.

-. (coords.), 2011. Politicas, redes y tecnologías en la comunicación para el de sarrollo. Zamora: Comunicación Social.

MARTínez NiCOLÁs, M., 2006. Masa (en situación) crítica. La investigación sobre periodismo en España: comunidad científica e intereses de conocimiento. Análisi. Quaderns de comunicació i cultura, 21, pp. 101-120.

- , 2008. La investigación de la comunicación en España: Apuntes sobre la emergencia, consolidación y desarrollo de una comunidad científica. En: Actas y memoria final del Congreso internacional fundacional AE-IC "I+C Investigar la Comunicación". Santiago de Compostela, 30-31 de enero y 1 de febrero de 2008.

Martínez Nicolás, M. y SAPERAs LAPIEdRA, E., 2011. La investigación sobre Comunicación en España (1998-2007): Análisis de los artículos publicados en revistas científicas. Revista Latina de Comunicación Social, 66, pp. 101-129. 
Mefalopulos, P., 2008. Development Communication Sourcebook. Broadening the Boundaries of Communication. Washington DC: The World Bank.

Melkote, S., 1991. Communication for development in the Third World: theory and practice. New Delhi [etc.]: Sage.

Miguel de Bustos, J. C., 2007. Comunicación Sostenible y Desarrollo Humano en la Sociedad de la Información. Madrid: AECI.

Moragas, M. de, 1981. Teorías de la comunicación. Investigaciones sobre medios en América y en Europa. Barcelona: Gustavo Gili.

- (ed.), 1985. Sociología de la comunicación de masas. Escuelas y autores. Barcelona: Gustavo Gili.

$\longrightarrow$, 1988. Los estudios sobre comunicación y nuevas tecnologías en España: indicaciones sobre sus antecedentes y estado actual. C.IN.CO: cuadernos de investigación en comunicación, 1, pp. 11-19. Madrid: Asociación para la Investigación Aplicada en Comunicación (AICE).

— 1990 . Delante de los negocios, detrás de los acontecimientos: nuevos problemas de la sociología de la comunicación de masas en España, 1986-1990. Telos, 22, pp. 58-64. Madrid: FUNDESCO.

Moragas, M. de y Corominas, M., 1988. La comunicació local a Catalunya (1975-1988). Barcelona: Diputació de Barcelona

MurCiano, M., 1980. Comunicación de masas, desarrollo y dependencia: economía, sociología y política en la investigación de la comunicación masiva en América Latina. Tesina para la obtención de la licenciatura en Ciencias de la Información por la Universidad Autónoma de Barcelona (UAB). Bellaterra: UAB.

PIÑUel, J. L., 2011. La docencia y la investigación universitarias en torno a la comunicación como objeto de estudio en Europa y América Latina. Colección Cuadernos Artesanos de Latina, 15. La Laguna, Tenerife: Sociedad Latina de Comunicación Social.

Prado, E., 1983. Las radios libres. Teoría y práctica de un movimiento alternativo. Barcelona: Mitre.

RaU de Almeida Callou, M., 2010. La comunicación para el desarrollo en Latinoamérica: evolución del paradigma y perspectivas actuales (1950-2010). Tesis doctoral presentada en el Departamento de Periodismo y Ciencias de la Comunicación de la Universidad Autónoma de Barcelona. Bellaterra: UAB.

Rodrigo Alsina, M., 2001. Teorías de la comunicación: ámbitos, métodos y perspectivas. Bellaterra [etc.]: Servei de Publicacions, Universitat Autònoma de Barcelona.

Rodrigo AlsinA, M. y GARCíA JimÉneZ, L., 2008. Teoría e investigación de la comunicación en España: Notas para un debate. En: Actas y memoria final del Congreso internacional fundacional AE-IC "I+C Investigar la Comunicación”. Santiago de Compostela, 30-31 de enero y 1 de febrero de 2008.

SÁEz BAEzA, Ch. y Barranquero, A., 2012. Trayectorias de la investigación académica sobre comunicación alternativa y comunicación para el cambio social en la universidad española. Ponencia presentada en el III Congreso Internacional AE-IC "Comunicación y Riesgo”. Tarragona, 18-20 de enero de 2012.

SAMPEDRO Blanco, V. (ed.), 2005. 13-M: multitudes on line. Madrid: Los Libros de la Catarata.

SERVAES, J., 1999. Communication for development: one world, multiple cultures. Cresskill (New Jersey): Hampton Press.

SIERRA, F. (coord.), 1997. Comunicación e insurgencia: La información y la propaganda en la Guerra de Chiapas. Hondarribia, Guipuzcoa: Hiru.

— 2000. Introducción a la teoría de la comunicación educativa. Sevilla: Editorial MAD. 
2006. Políticas de Comunicación y Educación. Barcelona: Gedisa.

2002. Comunicación, Educación y Desarrollo. Sevilla: Comunicación Social.

Soriano, J., 2008. El efecto ANECA. En Actas y memoria final del Congreso internacional fundacional AE-IC "I+C Investigar la Comunicación”. Santiago de Compostela, 30-31 de enero y 1 de febrero de 2008.

Valle Rojas, C. del, 2005. Comunicación para la participación: discursos, estructuras de medios y acceso en las experiencias participativas (gubernamentales y no gubernamentales) de Chile. Tesis doctoral presentada en el Departamento de Periodismo I de la Universidad de Sevilla. Sevilla: US.

Vidal Beneyto, J. (ed.), 1979. Alternativas populares a las comunicaciones de masas. Madrid: Consejo Superior de Investigaciones Sociológicas.

\title{
RESUMEN
}

Los estudios de comunicación para el desarrollo y el cambio social cuentan con medio siglo de existencia en el ámbito académico internacional. Razones de índole histórico-política y factores contextuales explican su tardía recepción en España, ya en los años ochenta, así como la marginal atención que les ha prestado nuestra academia hasta la primera década de los años 2000. Este artículo ofrece una visión panorámica e histórica de la evolución de la investigación y la docencia universitarias sobre comunicación para el desarrollo y el cambio social en nuestro país. Se examinan las circunstancias históricas y las condiciones que a lo largo de tres décadas han ido marcando el desarrollo de este campo disciplinar en España y se aportan elementos interpretativos adicionales que ayudan a entender el alcance y las limitaciones de este proceso. Finalmente, se apuntan las oportunidades y los desafíos que afronta su consolidación.

Palabras clave: Comunicación, desarrollo, cambio social, investigación sobre comunicación, docencia universitaria en comunicación, España.

\begin{abstract}
The studies in Communication for Development and Social Change have half a century of existence in the international scholarly field. In Spanish communication studies, their delayed development, starting in the 80's, and the poor attention that they have deserved in Spanish scholar community, not accomplished until the first decade of 2000, can both be explained in terms of some historical and political reasons, as well as with the context features. This paper offers a general overview, and an historical point of reflection, of the evolution of university teaching and research activities in Communication for Development and Social Change in our country. We examine the historical circumstances and conditions which, along three decades, have defined the development of this discipline in Spain, and we furnish some additional elements which help to the comprehension of the range and the limitations of the process. Finally, we indicate the opportunities and the challenges that the consolidation of this scholar development implies.
\end{abstract}

Key words: communication, development, social change, communicative research, university teaching in communication in Spain. 


\section{RÉSUMÉ}

Les études de communication pour le développement et le changement social ont déjà une moitié de siècle d'existence académique internationale. Dans les études de Communication en Espagne ce développement a été retardé, commençant dans les années 80 , et arrivant à avoir une attention pas marginale seulement après les premiers ans de la décade des 2000. Cet article nous offre une vision générale, et historique, de l'évolution de l'enseignement et la recherche scientifique dans la Communication pour le Développement et le Changement Social dans notre pays. On examine les circonstances historiques et les conditions que, au cours de trois décades, ont défini le développement de la discipline en Espagne, ainsi que on propose quelques éléments additionnais que aident à comprendre la portée et les limitations de ce procès. Finalement, on indique les opportunités et les défis que la consolidation de ce développement implique.

Mots clé: communication, développement, changement social, recherche sur communication, enseignement universitaire en communications en Espagne. 